\title{
Anisotropy of the $L_{2,3}$ x-ray magnetic linear dichroism of Fe films on GaAs: Experiment and $a b$ initio theory
}

\author{
Frithjof Nolting \\ Swiss Light Source, Paul Scherrer Institut, CH-5232 Villigen, Switzerland \\ Dominik Legut, Ján Rusz, and Peter M. Oppeneer \\ Department of Physics and Astronomy, Uppsala University, P.O. Box 516, S-751 20 Uppsala, Sweden
}

Georg Woltersdorf and Christian H. Back

Institut für Experimentelle und Angewandte Physik, Universität Regensburg, Universitätsstr. 31, D-93040 Regensburg, Germany

(Received 8 February 2010; revised manuscript received 27 August 2010; published 11 November 2010)

\begin{abstract}
We report investigations of the anisotropy of the x-ray magnetic linear dichroism (XMLD) of thin singlecrystalline Fe films on GaAs substrates with film thicknesses of $3 \mathrm{~nm}$ and $7 \mathrm{~nm}$, respectively. The orientation of the in-plane Fe magnetization with respect to the crystal axes is stepwise rotated from the [ $\left.\begin{array}{lll}1 & 1 & 0\end{array}\right]$ to the $\left[\begin{array}{ll}0 \\ 0\end{array}\right.$ $10]$ direction and the Fe $L_{2,3}$ XMLD spectrum is measured at every orientation step. A highly anisotropic XMLD signal at the Fe $L_{2}$ and $L_{3}$ edges is observed, which origin is analyzed in detail. We show that the measured anisotropic XMLD spectra are in reasonable agreement with ab initio calculated spectra. The XMLD anisotropy is shown to be a consequence of the cubic crystal-field split density of $3 d$ states, which are selectively probed by transitions from the spin-orbit and exchange-split $2 p$ core levels. We furthermore investigate computationally the influences of the spin-orbit interaction of the Fe $3 d$ valence electrons as well as of stress in the Fe films. The Fe $3 d$ spin-orbit interaction only leads to small modifications of the XMLD spectra, whereas tetragonal deformation of the films can cause a larger modification of the spectra.
\end{abstract}

DOI: 10.1103/PhysRevB.82.184415

PACS number(s): 78.20.Ls, 75.30.Gw

\section{INTRODUCTION}

$\mathrm{X}$-ray magneto-optical spectroscopy is an important tool for element selective studies of magnetic materials (see, e.g., Refs. 1-3 for recent reviews). The X-ray magnetic linear dichroism (XMLD) is a type of magnetic spectroscopy which has recently gained popularity. XMLD probes a magnetooptical response which - to lowest order-is quadratic in the atomic magnetization, whereas the $\mathrm{x}$-ray magnetic circular dichroism (XMCD) probes the response that is to lowest order linear in the atomic magnetization. XMLD offers therefore unique prospects for the element-selective investigation of antiferromagnets. Several XMLD investigations of antiferromagnetic materials (see, e.g., Refs. 4-11) as well as ferromagnetic materials ${ }^{12-17}$ have been reported.

The physical origin of XMLD differs in several essential aspects from the more commonly used XMCD. As has been known for a number of years, the XMCD exhibits a small dependence on the orientation of the magnetization in a crystalline material (see, e.g., Ref. 2). The small differences in the XMCD spectra measured for easy and hard magnetization axes have been related to the magnetocrystalline anisotropy of the orbital magnetic moment, which is a small quantity. $1,2,18$

A similar dependence on the magnetocrystalline anisotropy has been predicted also for $\mathrm{XMLD}^{19}$ but it is also a very small effect. ${ }^{15}$ A later theoretical investigation ${ }^{20}$ predicted that XMLD behaves in fact markedly different from

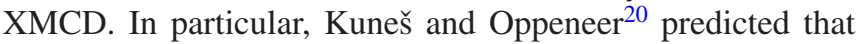
the XMLD depends strongly on the crystallographic orientation of the magnetization axes and as a consequence, a huge XMLD anisotropy was predicted for a $3 d$ atom in a cubic crystal field. It was shown ${ }^{20}$ that this anisotropy is a consequence of the core-level exchange splitting together with the cubic crystal-field split $3 d$ density of states (DOS), i.e., it arises from the spin-polarized part of the $e_{g}$ and $t_{2 g}$ states, which are selectively probed by transitions from the exchange-split $2 p$ core levels, while the direction of the magnetization defines the dipole allowed transitions. ${ }^{21}$ Later, a similar anisotropic XMLD has been observed for Mn-doped $\mathrm{GaAs}^{22}$ and for magnetic oxides ${ }^{9,11,23,24}$ and was also explained ${ }^{25,26}$ by the property of the cubic wave functions for the $3 d$ valance states with respect to the spin quantization axis. However, a direct experimental proof for itinerant $3 d$ metallic systems is still missing.

The huge anisotropy of the XMLD spectrum was originally predicted on the basis of $a b$ initio calculations for ferromagnetic $\mathrm{Fe}, \mathrm{Co}$, and $\mathrm{Ni}$ metals with cubic crystal symmetry. ${ }^{20}$ These density-functional-theory- (DFT) based calculations predicted, in particular, that for bcc $\mathrm{Fe}$ the XMLD spectrum for the [0 $\left.0 \begin{array}{ll}0 & 0\end{array}\right]$ magnetization axis would have a similar shape as the spectrum for the [ $\left[\begin{array}{lll}1 & 1 & 1\end{array}\right]$ magnetization axis, however, with an opposite sign. ${ }^{20,27}$ The purpose of the present paper is to probe the proposed anisotropy of the XMLD spectrum of Fe by performing measurements on single-crystalline Fe films. To this end, we have grown epitaxial $\mathrm{Fe}$ films on $\mathrm{GaAs}(001)$ and performed measurements of the XMLD in which the in-plane magnetization axis has been varied stepwise from the $\left[\begin{array}{lll}1 & 1 & 0\end{array}\right]$ to the $\left[\begin{array}{lll}0 & 1 & 0\end{array}\right]$ direction. For comparison, we have performed DFT $a b$ initio calculations of the XMLD spectrum of bcc Fe for magnetization directions corresponding to the experimental situation. Thus, we can compare directly measured and computed XMLD spectra as well as the anisotropy of the XMLD. In 
addition, we study Fe films of different thicknesses, which exhibit a volume fourfold magnetocrystalline anisotropy but with a uniaxial contribution due to the interface with the substrate. The direction of this uniaxial contribution is changing by $90^{\circ}$ resulting in a $90^{\circ}$ change in the effective easy axis and allows to investigate the influence of this property on the XMLD spectra.

\section{EXPERIMENT}

Epitaxial Fe films of $3 \mathrm{~nm}$ and $7 \mathrm{~nm}$ thickness were grown on $\operatorname{GaAs}(001)$ substrates using molecular beam epitaxy and capped with $2 \mathrm{~nm}$ of Au. Surface structure and chemical composition were studied in situ by low-energy electron diffraction and reflection high-energy electron diffraction. Details of the sample growth are published elsewhere. ${ }^{28}$ The effective anisotropy constants for the inplane fourfold anisotropy, $K_{1}$, and for the in-plane twofold anisotropy, $K_{u}$, were measured using ferromagnetic resonance. We find for the $3 \mathrm{~nm}$ Fe film $K_{1}=3.26$ $\times 10^{5} \mathrm{erg} / \mathrm{cm}^{3}$ and $K_{u}=-1.54 \times 10^{5} \mathrm{erg} / \mathrm{cm}^{3}$ and for the 7 $\mathrm{nm} \quad \mathrm{Fe}$ film $K_{1}=3.58 \times 10^{5} \mathrm{erg} / \mathrm{cm}^{3}$ and $K_{u}=1.62$ $\times 10^{5} \mathrm{erg} / \mathrm{cm}^{3}$, respectively. Due to the superposition of the uniaxial anisotropy with the fourfold anisotropy the easy axes have an angle of approximately $30^{\circ}$ and $150^{\circ}$ with respect to each other, as illustrated in the inset of Fig. 1. This results for the $3 \mathrm{~nm} \mathrm{Fe}$ in an effective uniaxial easy axis along the $\left[\begin{array}{lll}1 & 1 & 0\end{array}\right]$ axis and a hard axis along the $\left[\begin{array}{lll}-1 & 1 & 0\end{array}\right]$ direction while for the $7 \mathrm{~nm} \mathrm{Fe}$ film the effective uniaxial easy axis is along the $\left[\begin{array}{lll}-1 & 1 & 0\end{array}\right]$ direction and the hard axis along the $\left[\begin{array}{lll}1 & 1 & 0\end{array}\right]$ direction. This was confirmed by magnetooptical Kerr effect measurements and the effective easy axis coercivity was determined to be 30 Oe for both films and the hard axis saturation field to be 100 Oe for the $7 \mathrm{~nm}$ Fe film and 200 Oe for the $3 \mathrm{~nm} \mathrm{Fe}$ film. This change in the anisotropy can be explained by the competition between the $\mathrm{Fe}$ volume fourfold anisotropy and the uniaxial anisotropy originating at the $\mathrm{Fe} / \mathrm{GaAs}(001)$ interface ${ }^{28}$ modified by the induced strain of the Au capping layer. ${ }^{29}$

The polarization dependent $\mathrm{x}$-ray absorption spectra (XAS) were measured at beamline 4.0.2 of the Advanced Light Source. ${ }^{30}$ Circularly polarized x-rays (with about $80 \%$ degree of polarization) can be employed for XMCD measurements and for XMLD measurements $(99 \pm 1) \%$ linearly polarized x-rays can be used, the polarization plane of which can be orientated arbitrarily between $0^{\circ}$ and $90^{\circ}$. An eightpole vector magnet allowed applying magnetic fields of up to $0.8 \mathrm{~T}$ in any direction. ${ }^{31}$ All spectra were measured in the total electron yield mode by detecting the sample drain current. The sample can be rotated azimuthally around the surface normal allowing one to adjust the in-plane orientation of the sample. To this end hysteresis loops were measured in grazing incidence of $30^{\circ}$ with the magnetic field parallel to the circularly polarized x-rays, see Fig. 1 . The angle $\phi=0^{\circ}$ corresponds to the $\left[\begin{array}{lll}1 & 1 & 0\end{array}\right]$ direction which for the $7 \mathrm{~nm} \mathrm{Fe}$ film is the hard axis while for the $3 \mathrm{~nm}$ Fe film it is the easy axis. Note that the hysteresis loops are not corrected for the polarization degree and the angle of incidence.

Measured XAS of the 7 and $3 \mathrm{~nm}$ Fe films are shown in Fig. 2. Note that for comparison both spectra have been nor-
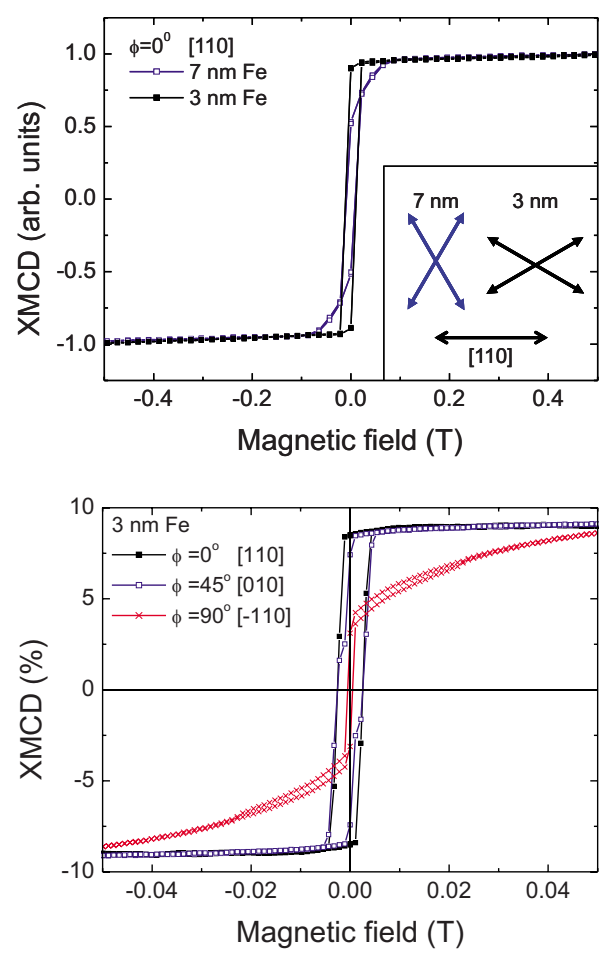

FIG. 1. (Color online) Hysteresis loops measured using the XMCD response for Fe films on $\mathrm{GaAs}(001)$. Top: comparison of the hysteresis loops of the 7-nm and 3-nm-thick Fe films measured with $\phi=0^{\circ}$ corresponding to the $\left[\begin{array}{lll}1 & 1 & 0\end{array}\right]$ axis which for the $7 \mathrm{~nm} \mathrm{Fe}$ film is the hard axis and for the $3 \mathrm{~nm}$ Fe film the effective uniaxial easy axis. Both films are fully saturated at $0.55 \mathrm{~T}$. In the inset the easy axes directions with respect to the $\left[\begin{array}{lll}1 & 1 & 0\end{array}\right]$ axis are shown. Bottom: hysteresis loops of the $3 \mathrm{~nm}$ Fe film measured for different in-plane orientations of the sample which shows the effective uniaxial anisotropy.

malized to unity at the maximum of the $L_{3}$ edge. The x-ray absorption spectrum of the $3 \mathrm{~nm}$ Fe film displays a small shoulder at the $\mathrm{Fe} L_{3}$ edges (at $710 \mathrm{eV}$ ), which indicates a slight oxidation. The $7 \mathrm{~nm}$ Fe film does not show such sign of oxidation, however, some oxidation cannot be completely excluded. The oxidation does not affect the magnetic anisotropies. The quality of the $7 \mathrm{~nm}$ film is good enough for qualitative comparisons.

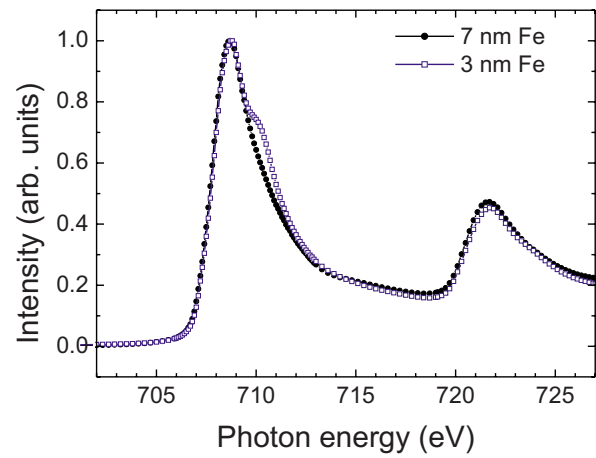

FIG. 2. (Color online) Measured XAS at the Fe $L$ edge for the 3-nm and 7-nm-thick Fe films, respectively. Note that for comparison both spectra have been normalized to unity at the maximum of the $L_{3}$ edge. 

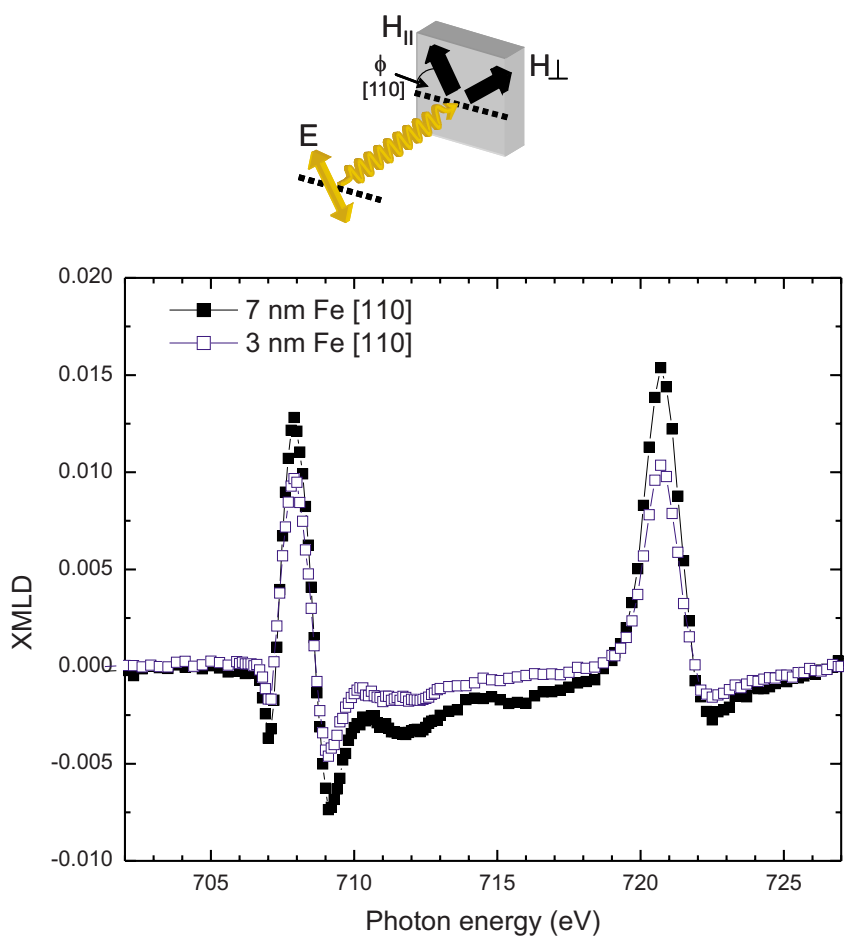

FIG. 3. (Color online) Top: sketch of the geometry used to measure the XMLD. The $L$-edge absorption spectra are measured for the magnetic field $\boldsymbol{H}$ applied parallel and perpendicular, to the X-ray polarization vector $\boldsymbol{E}$. Bottom: comparison of the XMLD spectra measured at $\phi=0^{\circ}$ for the $3 \mathrm{~nm}$ Fe film and for the $7 \mathrm{~nm} \mathrm{Fe}$ film.

The XMLD spectra were measured in normal incidence with an applied magnetic field of $0.55 \mathrm{~T}$ which was aligned a few degrees away from the sample plane. This small angle was chosen in order to increase the electron yield signal while it was verified that this procedure did not affect the XMLD spectra. The XMLD spectra were obtained by measuring the absorption spectra with the magnetic field $\boldsymbol{H}$ parallel, as well as perpendicular to the $\boldsymbol{E}$ vector of the linearly polarized light (see top panel of Fig. 3). ${ }^{23}$ The XMLD spectra plotted in the bottom panel of Fig. 3 have been obtained from the normalized XMLD asymmetry

$$
A=I\left(H_{\|}\right)-I\left(H_{\perp}\right) /\left[I\left(H_{\|}\right)+I\left(H_{\perp}\right)\right] .
$$

The spectral shape of the XMLD spectra of the 3 and $7 \mathrm{~nm}$ films is identical but the signal of the $3 \mathrm{~nm}$ film is slightly smaller. This can be a thickness-related saturation effect. The same XMLD results were obtained when instead of switching the magnetic field the polarization is reversed. In the following, all shown XMLD spectra have been obtained by switching the magnetic field. For the angular dependence, the $\boldsymbol{E}$ vector and the magnetic field direction were rotated simultaneously.

\section{AB INITIO THEORY OF THE XMLD}

\section{A. Computational framework for XMLD}

The theoretical foundation for $a b$ initio calculation of the XMLD has been outlined previously. ${ }^{20}$ We perform DFT calculations of the dielectric tensor, using the Kubo linear- response theory. The XMLD spectrum can be expressed as the difference in absorption of x-rays with $\boldsymbol{E} \| \boldsymbol{H}$ and $\boldsymbol{E} \perp \boldsymbol{H}$, which can be rewritten as a difference of the dielectric tensor elements $\varepsilon_{i j}$ that are selected through the orientation of the magnetization axis in the crystal and the light's polarization vector. ${ }^{32}$ In the earlier work of Kuneš and Oppeneer ${ }^{20}$ the magnetization axis was chosen along a cubic crystal axis having at least threefold symmetry (i.e., the [ $\left[\begin{array}{lll}0 & 0 & 1\end{array}\right]$ and $\left[\begin{array}{ll}1 & 1\end{array}\right.$ 1] axes). Under this condition, the dielectric tensor adopts a simpler form, as four off-diagonal tensor elements vanish (e.g., for $\boldsymbol{M}$ parallel to $\left.z=\left[\begin{array}{lll}0 & 0 & 1\end{array}\right]\right)$, the elements $\varepsilon_{x z}, \varepsilon_{z x}, \varepsilon_{y z}$, and $\varepsilon_{z y}$ vanish). The situation where the magnetization is oriented along a general, nonhigh symmetry axis is more complicated. For such a situation all the dielectric tensor elements are in general nonzero and have to be computed. More importantly, the expression for the XMLD signal in terms of the tensor elements changes. In order to obtain the eigenmodes for the light's propagation in the material, the full Fresnel equation has to be solved numerically, for the light's polarization vector parallel and normal to the occurring magnetization vector. ${ }^{32}$ Subsequently, the XMLD signal is computed from the difference of the absorptions $I\left(H_{\|}\right)$and $I\left(H_{\perp}\right)$ [Eq. (1)]. The information on the reduced symmetry due to the particular magnetization direction in the cubic crystal is thus comprised in the dielectric tensor and is transferred to the XMLD.

For the numerical evaluation of the dipole matrix elements and the DFT single-particle energies that are required for calculation of the dielectric tensor elements we used the full-potential, linearized augmented plane-wave (LAPW) method as implemented in the WIEN2K code. ${ }^{33}$ The wave functions are expanded into products of spherical harmonics $Y_{l m}$ and radial wave functions within a given atomic sphere. The core states are obtained as solutions of the Kohn-ShamDirac equation; for the core potential we adopt the spherical approximation. The effect of the exchange field on the core levels is included as outlined in Refs. 34 and 35. The relativistic valence states are computed within the full, nonspherical potential, using the second variational approach to include self-consistently the spin-orbit (SO) interaction. ${ }^{36} \mathrm{In}$ the present WIEN2K implementation local orbitals are included in the basis, and the LAPW basis set has been used. The electronic structure calculations for bcc $\mathrm{Fe}$ are performed with $2431 k$ points in the irreducible Brillouin zone (1/4th). As we are dealing with a general magnetic quantization axis along nonhigh-symmetry directions, only four symmetry operations remain (viz., $E, I, C_{2}$, and $\sigma_{h}$ ). The atomic sphere radius $\left(R_{m t}\right)$ of $\mathrm{Fe}$ has been set to $2.2 \mathrm{Bohr}$ radii. Approximately 85 basis functions per atom have been used. The product of $R_{m t}$ and maximum reciprocal space vector $\left(k_{\max }\right)$, i.e., the cut-off energy $\left(R K_{\max }\right)$ is set to 8.5 and the largest reciprocal vector $\boldsymbol{G}$ in the charge Fourier expansion, $G_{\max }$, is equal to 14 . The convergence of the total energy is better than $1 \times 10^{-5} \mathrm{Ry} /$ atom.

\section{B. Anisotropy of the XMLD in cubic crystal fields}

Theoretical models for the anisotropy of the XMLD have been given by van der Laan ${ }^{19}$ and by Kuneš and Oppeneer. ${ }^{20}$ 
The latter model, ${ }^{20}$ which has become the accepted model for XMLD anisotropies of magnetic oxides, ${ }^{25,26}$ will be employed here in the interpretation of our data. In short, in this model the XMLD is related to the exchange splitting of the $2 p$ core levels and the influence of the crystal field. Without splitting of the core levels due to the exchange field, the XMLD would practically vanish, (i.e., it would only be caused by the anisotropy of the SO interaction in the valence states). Similarly, there would be a vanishing XMLD anisotropy in the absence of the crystal field. In the presence of the cubic crystal field the XMLD signal of the transition metal atom is related to the spin-polarized $e_{g}$ and $t_{2 g}$ states that are selectively probed for a given geometry. As a consequence, the XMLD asymmetry $A$ in cubic systems can be expressed as energy derivatives of the $e_{g}$ and $t_{2 g} 3 d$-partial $\operatorname{DOS}^{20,27}$

$$
A \propto \Delta \frac{d}{d E}\left\{\alpha\left(t_{2 g \uparrow}-t_{2 g \downarrow}\right)(E)+\beta\left(e_{g \uparrow}-e_{g \downarrow}\right)(E)\right\},
$$

where $\Delta$ is the exchange splitting of the core levels, $t_{2 g \uparrow}(E)$ is the energy-dependent majority spin $t_{2 g}$ partial DOS, and $\alpha$ and $\beta$ are numerical constants that depend on the direction of the magnetization in the crystal. For the $\left[\begin{array}{lll}0 & 0 & 1\end{array}\right]$ and $\left[\begin{array}{lll}1 & 1 & 1\end{array}\right]$ magnetization directions $\alpha$ is $-1,1$ and $\beta=2,-1$, respectively. This expression can be generalized to arbitrary magnetization directions in the crystal. ${ }^{37}$ Hence, the XMLD spectrum of a transition-metal atom in a cubic crystal field is always given by the sum of two independent $e_{g}$ and $t_{2 g}$ contributions and, depending on the direction of the magnetization axis in the crystal, different combinations of the spinpolarized $e_{g}$ and $t_{2 g}$ states are probed in XMLD experiments. Two effects are neglected in the derivation of Eq. (2), the influences of tetragonal distortion and that of SO interaction in the valence states. Below their importance for the angledependent XMLD spectra will be assessed by $a b$ initio calculations and by comparing the angle-dependent XMLD spectra of the Fe films with the two different orientations of the effective uniaxial anisotropy.

\section{RESULTS}

\section{A. $7 \mathrm{~nm}$ Fe on GaAs(001)}

For the comparison with the above described $a b$ initio theory of the XMLD we start with the $7 \mathrm{~nm}$ Fe film having the effective uniaxial anisotropy along the $\left[\begin{array}{lll}-1 & 1 & 0\end{array}\right]$ axis. The sample is rotated azimuthally, probing the $x y$ plane. For clarity, we first plot the measured XMLD spectra in $15^{\circ}$ steps from $0^{\circ}$ to $90^{\circ}$ in Fig. 4 . The largest difference of the XMLD spectra can be seen between the $0^{\circ}$ and $45^{\circ}$ orientations, reflecting the cubic symmetry of the epitaxial film but not the effective uniaxial magnetic anisotropy. Notably, the XMLD spectra of the $L_{3}$ edge and $L_{2}$ edge show very different characteristics. The spectra at the $L_{2}$ edge consist of two peak structures, at 721 and $722 \mathrm{eV}$, which are inverting their sign as can be seen when comparing the $0^{\circ}$ and $45^{\circ}$ spectra. In contrast, the $L_{3}$ edge shows a much more complex behavior and displays for each angle a unique spectrum. This is a similar behavior as recently reported for the XMLD aniso-

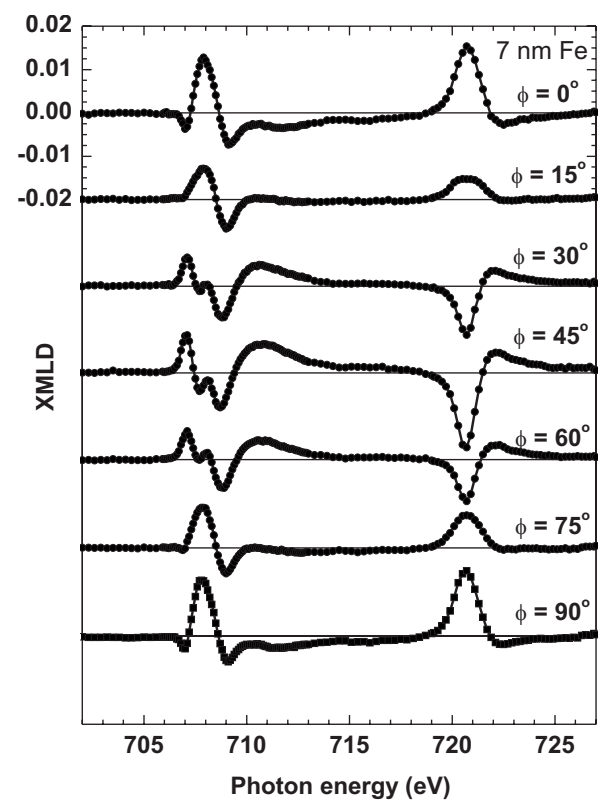

FIG. 4. Angular dependence of the Fe $L_{3,2}$ XMLD spectrum for $7 \mathrm{~nm} \mathrm{Fe} / \mathrm{GaAs}(001)$, measured in $15^{\circ}$ steps. The XMLD spectra are obtained as the difference between two absorption spectra recorded with the magnetic field $\boldsymbol{H}$ parallel and perpendicular to the x-ray polarization vector $\boldsymbol{E}$ (see Fig. 3). $\phi$ corresponds to the angle between the polarization vector and the $\left[\begin{array}{lll}1 & 1 & 0\end{array}\right]$ crystal direction. The most pronounced difference can be observed between the 0 and $45^{\circ}$ XMLD spectra.

tropy of $\mathrm{Ni}^{2+} \cdot{ }^{24}$ For a more detailed comparison, the measured XMLD spectra are plotted in Fig. 5 in $5^{\circ}$ steps from $0^{\circ}$ to $50^{\circ}$. These XMLD spectra for finer angular steps reveal that a multipeak structure occurs at the $L_{3}$ edge, consisting of three distinct peaks. These peaks occur at energies of about 707, 708, and $709 \mathrm{eV}$, and display each a distinct angular dependence. The first peak at $707 \mathrm{eV}$ is small at $0^{\circ}$, vanishes with increasing angle near $\phi=15^{\circ}$ where after it increases with reversed sign to reach a maximum at $45^{\circ}$. The second peak at $708 \mathrm{eV}$ attains its maximum at $0^{\circ}$, diminishes with increasing angle and also becomes sign reversed. The third peak at $709 \mathrm{eV}$ does not display a notable angular dependence. This peak has a negative sign and its size remains constant independent of $\phi$. We note, for later discussion, that this peak occurs close to multiplet energy associated with possible oxidation of the film. The angle-dependent XMLD spectra in Fig. 5 furthermore reveal a broad hump at the high-energy side of the $L_{3}$ peak, extending for some angles from 710 to $713 \mathrm{eV}$. This hump also inverts its sign, from a maximum at $45^{\circ}$ to a minimum at $0^{\circ}$. A similar hump cannot be discerned at $L_{2}$ edge and neither does an angleindependent peak appear at the $L_{2}$ edge.

To simulate the measurements we performed calculations of XAS spectra for bulk body centered cubic iron while gradually rotating the magnetic polarization vector as in the experiment, from [ $\left[\begin{array}{lll}1 & 1 & 0\end{array}\right]$ through $\left[\begin{array}{lll}0 & 1 & 0\end{array}\right]$ to $\left[\begin{array}{lll}-1 & 1 & 0\end{array}\right]$. The calculated XMLD spectra are shown in Fig. 6. Note that the energies are given relative to the onset of the $L_{3}$ edge; $0 \mathrm{eV}$ corresponds to about $707 \mathrm{eV}$ in the measured spectra. The calculations were performed either with (solid line) or with- 


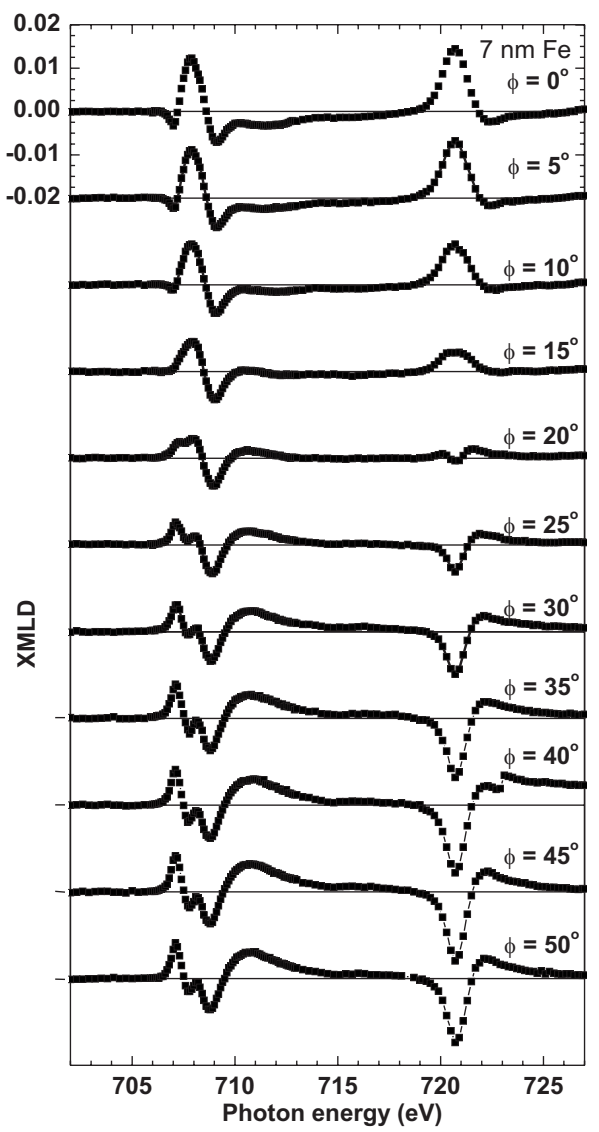

FIG. 5. Detailed angular dependence of the Fe $L_{3,2}$ XMLD spectra for $7 \mathrm{~nm} \mathrm{Fe} / \mathrm{GaAs}(001)$, measured in $5^{\circ}$ steps.

out (dashed line) SO interaction in the valence band. Only a small difference between the two spectra is observed, demonstrating the weak influence of the valence-band SO interaction on the spectral shape. A similar result was previously reported for the XMLD spectrum with magnetization vector oriented along a high-symmetry axis in a cubic crystal. ${ }^{20}$

The calculations are in reasonable qualitative agreement with the measurements. To start with, the magnitude of the computed XMLD is on the order of $1-2 \%$, in good agreement with the measured amplitudes. The following major spectral characteristics are observed: (i) a maximal change occurring between the $0^{\circ}$ and $45^{\circ}$ spectra, observed most clearly at the $L_{2}$ edge, (ii) a multipeak structure at the $L_{3}$ edge for specific angles, and (iii) an approximate inversion of the shape of the XMLD spectrum for angles $0^{\circ}$ and $45^{\circ}$. Because of the fourfold symmetry in the $x y$ plane, the spectra for angles $\phi$ smaller than $45^{\circ}$ are identical to those for angles $90^{\circ}-\phi$, i.e., $\operatorname{XMLD}\left(45^{\circ}+\phi\right)=\operatorname{XMLD}\left(45^{\circ}-\phi\right)$. Note that this property follows from the $a b$ initio calculation and is not enforced through any symmetry constraint. Both the calculated and experimental $L_{2}$ XMLD spectra have the feature that the $\left[\begin{array}{lll}1 & 1 & 0\end{array}\right]\left(\phi=0^{\circ}\right)$ and $\left[\begin{array}{lll}0 & 1 & 0\end{array}\right]\left(\phi=45^{\circ}\right)$ spectra are very similar to one another but sign reversed. Looking now closer at the $L_{3}$ edge, we can observe two clear peaks (at 1 and 2 $\mathrm{eV}$ ) which invert their sign with evolving angle. There is also a prepeak at $0 \mathrm{eV}$, which changes with $\phi$ but does not reverse its sign. This might however be related to the presence of a nearby peak combined with lifetime broadening. A clear dif-

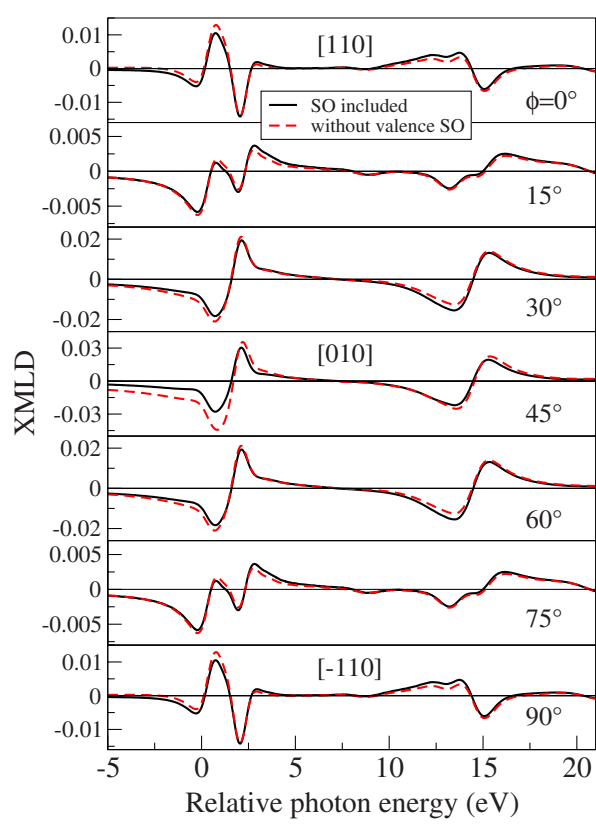

FIG. 6. (Color online) Ab initio calculated angular dependence of the XMLD spectrum of Fe. The orientation the magnetization vector in the crystal is rotated from the $\left[\begin{array}{lll}1 & 1 & 0\end{array}\right]$ to the $\left[\begin{array}{lll}-1 & 1 & 0\end{array}\right]$ direction. The spectra are computed with (full curve) and without (dashed curve) the spin-orbit interaction of the valence states. Note that the energies are given relative to the onset of the $L_{3}$ edge and that $0 \mathrm{eV}$ corresponds to about $707 \mathrm{eV}$ in the measured spectra.

ference between the experimental and theoretical XMLD spectra is the appearance of the angle-invariant peak at 709 $\mathrm{eV}$ in the experimental spectrum, which does not appear in the $a b$ initio computed spectrum. A small difference between the calculation and the experiment is observed if one compares the absolute values. The change in the sign of the XMLD spectrum at the $L_{2}$ edge occurs in the experiment around $20^{\circ}$, as is apparent from the more detailed scan in Fig. 5, while in the theoretical spectra the sign reversal is closer to $15^{\circ}$. However, this discrepancy is near to the experimental uncertainty of a few degrees. Further differences can be seen in the details of the spectral shape. The ratio of the XMLD at the $L_{3}$ and the $L_{2}$ edge is close to one in the experiment with at some angles even a larger signal appearing at the $L_{2}$ edge. In the calculation the XMLD at the $L_{3}$ edge is always larger than at the $L_{2}$ edge. The experiment moreover displays the aforementioned broad hump at the high-energy side of the $L_{3}$ edge, where the calculations show only a smaller tail.

The origin for these quantitative discrepancies is not known, and further investigations are needed in order to determine whether the calculations can be improved by including effects beyond the ground-state electronic structure, such as effects of the core hole and its dynamical screening. Tentatively, the appearance of the hump could be attributed to a core-hole effect which is not included in the calculations. Alternatively, imperfections of the sample such as strain or oxidation may also affect the measured XMLD to some extent. The influence of oxidation is indicated by the appearance of the angle-independent peak in the measured XMLD, near to the energy where an oxidation-related multiplet re- 


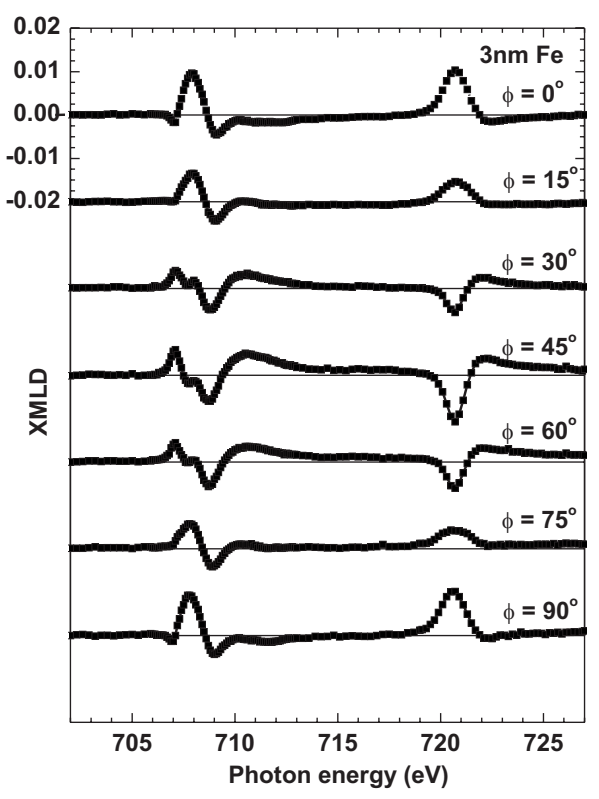

FIG. 7. Angular dependence of the Fe $L_{3,2}$ XMLD spectra for 3 $\mathrm{nm} \mathrm{Fe} / \mathrm{GaAs}(001)$, measured in $15^{\circ}$ steps. The XMLD spectra are obtained in the same way as those for $7 \mathrm{~nm} \mathrm{Fe} / \mathrm{GaAs}(001)$. $\phi$ corresponds to the angle between the polarization vector and the [1 1 $0]$ direction. The spectra are almost identical to the spectra of $7 \mathrm{~nm}$ Fe/GaAs(100), shown in Fig. 4.

sponse appears. The conclusion regarding strain is in part supported by our results on the $3 \mathrm{~nm}$ Fe film, where we find that the lattice mismatch between the GaAs substrate and $\mathrm{Fe}$ film must be taken into account. Distortions from the bcc structure through strain lead to significant modifications of the calculated XMLD, as will be shown below.

\section{B. $3 \mathrm{~nm}$ Fe on $\operatorname{GaAs}(001)$}

The $3 \mathrm{~nm} \mathrm{Fe}$ film has a $90^{\circ}$-rotated effective uniaxial anisotropy compared to the $7 \mathrm{~nm} \mathrm{Fe}$ film and one might expect to observe a different anisotropy of the XMLD. However, the angular dependence of the XMLD, plotted in Fig. 7, is almost identical to that of the $7 \mathrm{~nm} \mathrm{Fe}$ film. A maximal change in the XMLD spectra is again seen between the $0^{\circ}$ and $45^{\circ}$ orientations and the spectra for angles $\phi$ smaller than $45^{\circ}$ are identical to angles $90^{\circ}-\phi$. At the $L_{3}$ edge a multipeak structure appears while the $L_{2}$ edge spectrum consists essentially of a peak and a minimum, a structure which is changing its sign around $20^{\circ}$. Comparing the angular dependence of the XMLD of the 3 and $7 \mathrm{~nm}$ film no indication of a uniaxial behavior can be found. This implies that the difference in the magnetic anisotropy of the two and even the difference of hard and easy axes is not reflected in the anisotropy of the XMLD. The extent of the similarity of the XMLD spectra of the two films can be seen in Fig. 3, where the corresponding spectra are plotted together. The XMLD of the $3 \mathrm{~nm}$ film is smaller, as mentioned earlier, but it can be scaled on that of the $7 \mathrm{~nm}$ film. After scaling a difference in the spectra is observed only in the energy range of 710-713 $\mathrm{eV}$. This difference is probably related to the slight oxidation of the $3 \mathrm{~nm} \mathrm{Fe}$ film (see the XAS in Fig. 2) and not to the

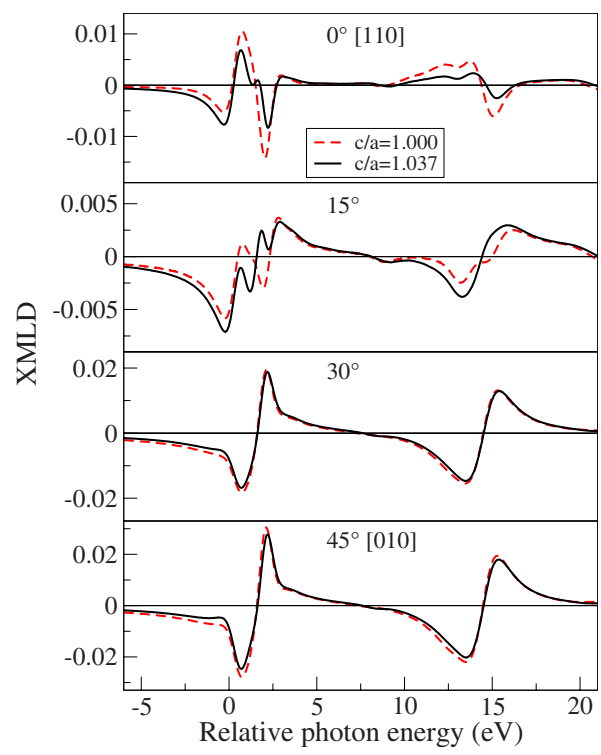

FIG. 8. (Color online) Ab initio computed angular dependent XMLD spectra of tetragonally distorted Fe with $c / a=1.037$ (full curve), compared to XMLD spectra without distortion $(c / a=1.000$, dashed curve).

magnetic anisotropy of the films. This finding confirms the $a b$ initio calculations ${ }^{20}$ which showed that the $3 d$ valenceband spin-orbit coupling only has a small influence on the anisotropic XMLD. Furthermore, since the $3 \mathrm{~nm}$ Fe film was slightly oxidized but the $7 \mathrm{~nm}$ film is expected to be less oxidized, the observed $L_{3}$ multipeaks in the XMLD of the 7 and $3 \mathrm{~nm}$ Fe cannot solely be due to oxidation of the film. Otherwise, one would expect a much stronger multipeak structure for the $3 \mathrm{~nm}$ Fe film. The presence of the angleinvariant peak in the $7 \mathrm{~nm}$ film XMLD, however, can be interpreted as an indication for a slight oxidation in this film as well.

Another possible explanation for the multipeak structures is a distortion of the film. In order to investigate its influence on the XMLD signal, we included the known lattice distortion of the Fe film in our calculations. The GaAs substrate deforms the cubic structure of $\mathrm{Fe}$ into a tetragonal one. In our calculations we considered the $c / a$ ratio of 1.037 , corresponding to the tetragonal deformation of an Fe overlayer on GaAs. ${ }^{38,39}$ The calculated XMLD spectra are shown in Fig. 8. Again, a qualitative reasonable agreement with the measurements is obtained. The inclusion of the distortion mainly leads to changes of the XMLD at the $L_{3}$ edge. The multipeak structure is modified and starts to appear also at other angles, e.g., $0^{\circ}$. The actual profile of the strain in the Fe film and the corresponding deformation is not known. Therefore a strain profile in the sample may account for a part of the observed difference between calculations and experiment with respect to the multipeak structure at the $L_{3}$ edge. We note, however, that the computed anisotropic XMLD of tetragonally distorted $\mathrm{Fe}$ does not contain an angle-invariant peak. Hence, the appearance of this peak cannot be explained by ab initio calculations for pure bulk Fe. This reinforces the interpretation that it is related to a slight oxidation. 


\section{CONCLUSIONS}

The angular dependence of the XMLD spectra of thin $\mathrm{Fe}$ films on $\mathrm{GaAs}(001)$ was measured for two systems, a $7 \mathrm{~nm}$ Fe film which has an effective uniaxial easy axis along the $\left[\begin{array}{lll}-1 & 1 & 0\end{array}\right]$ direction and a $3 \mathrm{~nm}$ Fe film where the effective uniaxial easy axis is rotated in-plane by $90^{\circ}$ toward the $\left[\begin{array}{ll}1 & 1\end{array}\right.$ 0] direction. Both systems are found to exhibit an almost identical characteristic of the angular dependence of the XMLD. The measured strong anisotropy of the XMLD was compared to $a b$ initio band-structure calculations of the XMLD spectra and a reasonable agreement with the major characteristics of the anisotropic XMLD spectra was observed. In particular, the XMLD spectra for the $\left[\begin{array}{lll}1 & 1 & 0\end{array}\right]$ and $[0$ $10]$ magnetization axes are found to have a very similar shape, especially at the $L_{2}$ edge, but the spectra for theses two directions show a reversed sign. The obtained agreement confirms the theoretical picture of XMLD. The shape of the XMLD spectrum depends on the $2 p$ core-state exchange splitting as well as the $3 d$-partial densities of states. In particular, the XMLD probes the spin-polarized unoccupied $e_{g}$ and $t_{2 g}$ final states, which leads to a strong dependence of the XMLD with respect to the magnetization axis and crystallographic orientation. If the crystal field would be absent, e.g., for an atom in spherical symmetry, the XMLD would not display the observed large anisotropy, only a very small anisotropy due the SO interaction would remain. ${ }^{20}$ In our XMLD measurements a different angular behavior of the $L_{3}$ and $L_{2}$ edges is observed. Whereas the $L_{2}$ XMLD peak structure shows mainly a reversal when the magnetization is stepwise rotated, the XMLD at the $L_{3}$ edge displays a more complicated behavior. Three distinct peaks can be discerned in the XMLD spectra. One of these is angle-invariant and is tentatively attributed to a slight oxidation of the Fe films. The other two peaks invert their sign with evolving angle, something which is also observed in the calculations in which this behavior depends on the strain of the system. Although the $7 \mathrm{~nm}$ and $3 \mathrm{~nm}$ film have a different magnetocrystalline anisotropy they have virtually the same XMLD anisotropy. This demonstrates that the spin-orbit interaction in the valence states is only a very minor modification of the XMLD, in agreement with the $a b$ initio band-structure calculations that predict that the influence of SO interaction in the valence band leads only to rather small changes in the XMLD spectra.

In summary, we demonstrated experimentally the angular dependence of the XMLD spectra on the magnetization axis for the itinerant ferromagnet Fe. The $a b$ initio calculations were performed for arbitrary magnetization angles in the crystal lattice through a complete numerical solution of the Fresnel equation and the effect of strain in the lattice has been taken into account. Reasonable qualitative agreement between the calculations-which incorporate the full spinpolarized band character of the system-and the measurement was obtained. This agreement allows a more detailed probing of itinerant ferromagnets with XMLD in the future. Also, our findings show the importance of the crystallographic orientation in order to properly analyze XMLD spectra.

\section{ACKNOWLEDGMENTS}

We thank Jan Kuneš and Jörg Raabe for helpful discussions and Elke Arenholz for the excellent help during the measurements at the ALS. This work has been supported by the Swedish Research Council (VR), by the European Community's seventh framework program EU-ITN "FANTOMAS," the G. Gustafsson Foundation, STINT, and the Swedish National Infrastructure for Computing (SNIC). The Advanced Light Source is supported by the Director, Office of Science, Office of Basic Energy Sciences, of the U.S. Department of Energy under Contract No. DE-AC02$05 \mathrm{CH} 11231$.
${ }^{1}$ J. Kortright, D. Awschalom, J. Stöhr, S. D. Bader, Y. Idzerda, S. S. P. Parkin, I. K. Schuller, and H.-C. Siegmann, J. Magn. Magn. Mater. 207, 7 (1999).

${ }^{2}$ J. Stöhr and H.-C. Siegmann, Magnetism: From Fundamentals to Nanoscale Dynamics (Springer, Berlin, 2006).

${ }^{3}$ H. A. Dürr, T. Eimüller, H.-J. Elmers, S. Eisebitt, M. Farle, W. Kuch, F. Matthes, M. Martins, H.-Ch. Mertins, P. M. Oppeneer, L. Plucinski, C. M. Schneider, H. Wende, W. Wurth, and H. Zabel, IEEE Trans. Magn. 45, 15 (2009).

${ }^{4}$ P. Kuiper, B. G. Searle, P. Rudolf, L. H. Tjeng, and C. T. Chen, Phys. Rev. Lett. 70, 1549 (1993).

${ }^{5}$ W. J. Antel, F. Perjeru, and G. R. Harp, Phys. Rev. Lett. 83, 1439 (1999).

${ }^{6}$ A. Scholl, J. Stöhr, J. Lüning, J. W. Seo, J. Fompeyrine, H. Siewart, J.-P. Locquet, F. Nolting, S. Anders, E. E. Fullerton, M. R. Scheinfein, and H. A. Padmore, Science 287, 1014 (2000).

${ }^{7}$ W. Zhu, L. Seve, R. Sears, B. Sinkovic, and S. S. P. Parkin, Phys. Rev. Lett. 86, 5389 (2001).

${ }^{8}$ P. M. Oppeneer, H.-Ch. Mertins, D. Abramsohn, A. Gaupp, W.
Gudat, J. Kuneš, and C. M. Schneider, Phys. Rev. B 67, 052401 (2003).

${ }^{9}$ S. Czekaj, F. Nolting, L. J. Heyderman, P. R. Willmott, and G. van der Laan, Phys. Rev. B 73, 020401(R) (2006).

${ }^{10}$ Y. Z. Wu, Y. Zhao, E. Arenholz, A. T. Young, B. Sinkovic, C. Won, and Z. Q. Qiu, Phys. Rev. B 78, 064413 (2008).

${ }^{11}$ I. P. Krug, F. U. Hillebrecht, M. W. Haverkort, A. Tanaka, L. H. Tjeng, H. Gomonay, A. Fraile-Rodríguez, F. Nolting, S. Cramm, and C. M. Schneider, Phys. Rev. B 78, 064427 (2008).

${ }^{12}$ M. M. Schwickert, G. Y. Guo, M. A. Tomaz, W. L. O'Brien, and G. R. Harp, Phys. Rev. B 58, R4289 (1998).

${ }^{13}$ J. B. Kortright and S.-K. Kim, Phys. Rev. B 62, 12216 (2000).

${ }^{14}$ H.-Ch. Mertins, P. M. Oppeneer, J. Kuneš, A. Gaupp, D. Abramsohn, and F. Schäfers, Phys. Rev. Lett. 87, 047401 (2001).

${ }^{15}$ S. S. Dhesi, G. van der Laan, E. Dudzik, and A. B. Shick, Phys. Rev. Lett. 87, 067201 (2001).

${ }^{16}$ H.-Ch. Mertins, S. Valencia, A. Gaupp, W. Gudat, P. M. Oppeneer, and C. M. Schneider, Appl. Phys. A: Mater. Sci. Process. 80, 1011 (2005). 
${ }^{17}$ W. Kuch, F. Offi, L. I. Chelaru, J. Wang, K. Fukumoto, M. Kotsugi, J. Kirschner, and J. Kuneš, Phys. Rev. B 75, 224406 (2007).

${ }^{18}$ D. Weller, J. Stöhr, R. Nakajima, A. Carl, M. G. Samant, C. Chappert, R. Mégy, P. Beauvillain, P. Veillet, and G. A. Held, Phys. Rev. Lett. 75, 3752 (1995).

${ }^{19}$ G. van der Laan, Phys. Rev. Lett. 82, 640 (1999).

${ }^{20}$ J. Kuneš and P. M. Oppeneer, Phys. Rev. B 67, 024431 (2003).

${ }^{21}$ The huge XMLD anisotropy has been called (Refs. 20 and 27) magnetocrystalline anisotropy of the XMLD to emphasize that it is the anisotropy related to the crystallographic orientation of the magnetization vector and not to express that it is related to the spin-orbit interaction. As was explicitly demonstrated in the first paper discussing (Ref. 20) the huge XMLD anisotropy, the SO interaction of the $3 d$ valence band gives only a very small contribution.

${ }^{22}$ A. A. Freeman, K. W. Edmonds, G. van der Laan, N. R. S. Farley, T. K. Johal, E. Arenholz, R. P. Campion, C. T. Foxon, and B. L. Gallagher, Phys. Rev. B 73, 233303 (2006).

${ }^{23}$ E. Arenholz, G. van der Laan, R. V. Chopdekar, and Y. Suzuki, Phys. Rev. B 74, 094407 (2006).

${ }^{24}$ E. Arenholz, G. van der Laan, R. V. Chopdekar, and Y. Suzuki, Phys. Rev. Lett. 98, 197201 (2007).

${ }^{25}$ G. van der Laan, E. Arenholz, R. V. Chopdekar, and Y. Suzuki, Phys. Rev. B 77, 064407 (2008).

${ }^{26}$ G. van der Laan and E. Arenholz, Eur. Phys. J. Spec. Top. 169, 187 (2009)

${ }^{27}$ J. Kuneš, P. M. Oppeneer, S. Valencia, D. Abramsohn, H.-Ch.
Mertins, W. Gudat, M. Hecker, and C. M. Schneider, J. Magn. Magn. Mater. 272, 2146 (2004).

${ }^{28}$ R. Urban, G. Woltersdorf, and B. Heinrich, Phys. Rev. Lett. 87, 217204 (2001).

${ }^{29}$ O. Thomas, Q. Shen, P. Schieffer, N. Tournerie, and B. Lépine, Phys. Rev. Lett. 90, 017205 (2003).

${ }^{30}$ A. T. Young, E. Arenholz, S. Marks, R. Schlueter, C. Steier, H. A. Padmore, A. P. Hitchcock, and D. G. Castner, J. Synchrotron Radiat. 9, 270 (2002).

${ }^{31}$ E. Arenholz, Rev. Sci. Instrum. 76, 083908 (2005).

${ }^{32}$ P. M. Oppeneer, in Handbook of Magnetic Materials, edited by K. H. J. Buschow (Elsevier, Amsterdam, 2001), Vol. 13, pp. 229-422.

${ }^{33}$ P. Blaha, K. Schwarz, G. K. H. Madsen, D. Kvasnicka, and J. Luitz, WIEN2K, Vienna University of Technology, 2001.

${ }^{34}$ J. Kuneš, P. M. Oppeneer, H.-Ch. Mertins, F. Schäfers, A. Gaupp, W. Gudat, and P. Novák, Phys. Rev. B 64, 174417 (2001).

${ }^{35}$ J. Kuneš, P. M. Oppeneer, H.-Ch. Mertins, F. Schäfers, A. Gaupp, W. Gudat, and P. Novák, J. Magn. Magn. Mater. 240, 454 (2002).

${ }^{36}$ J. Kuneš, P. Novák, M. Diviš, and P. M. Oppeneer, Phys. Rev. B 63, 205111 (2001).

${ }^{37}$ D. Legut, J. Rusz, and P. M. Oppeneer (unpublished).

${ }^{38}$ S. McPhail, C. M. Gürtler, F. Montaigne, Y. B. Xu, M. Tselepi, and J. A. C. Bland, Phys. Rev. B 67, 024409 (2003).

${ }^{39}$ R. A. Gordon, E. D. Crozier, D.-T. Jiang, P. S. Budnik, T. L. Monchesky, and B. Heinrich, Surf. Sci. 581, 47 (2005). 